\title{
Viel Bewegung in den Bereichen Mundspüllösungen und Lokalanästhesie
}

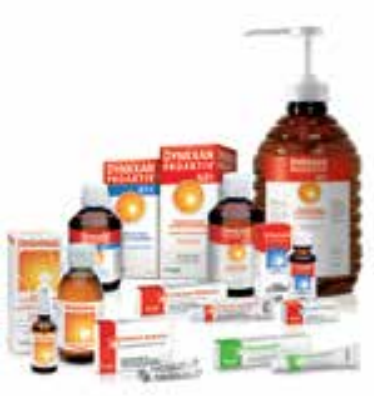

Auf der Pressekonferenz von Kreussler am 10. März 2015 anlässlich der IDS standen 2 facettenreiche Themenbereiche im Mittelpunkt: Mundspüllösungen und Lokalanästhesie. Zu den in der Zahnmedizin am häufigsten genutzten prophylaktischen und therapeutischen Agenzien gehört Chlorhexidindiglukonat (kurz CHX). Es gilt in seiner 0,2 \%igen Konzentration als effektiver Wirkstoff gegen Plaque und Gingivitis. Weniger spektakulär - dennoch erwähnenswert - ist auch die Tatsache, dass durch Einsatz 0,2\% chlorhexidinhaltiger Lösung oder ggfs. Gelen/Lacken in einer Konzentration von >1 \% MutansStreptokokken gezielt ausgeschaltet werden können. So kann das individuelle Kariesrisiko reduziert und eine Übertragung von Keimen (beispielsweise von der Mutter auf das Kind) minimieren werden. Grundsätzlich stellt die klassische Lokalanästhesie vor parodontalen Maßnahmen eine gute Möglichkeit dar, Schmerzen entgegenzuwirken. Eine attraktive Alternative ist die Anästhesie mit DYNEXAN Mundgel. Sie lässt sich dank der Applikation mit einer stumpfen Kanüle aus der klassischen Zylinderampulle in den Sulkus einfach durchführen. Das antiseptische Mund- und Rachentherapeutikum DYNEXAN PROAKTIV 0,2 \% CHX deckt das gesamte oben skizzierte Einsatzspektrum ab und geht konform mit den Leitlinien der Bundeszahnärztekammer. Bei DYNEXAN Mundgel handelt es sich um ein Monopräparat mit den bekannten Vorteilen in der Verträglichkeit - sogar ohne Altersbeschränkung verwendbar.

Nach einer Pressemitteilung der Chemische Fabrik Kreussler \& Co. GmbH,

Wiesbaden 